\title{
Selection of design structure and method of energy transformation in wind power plant
}

\author{
Andrey Pushkarev ${ }^{1, *}$, Dmitriy Khvorenkov ${ }^{1}$, Olga Varfolomeeva ${ }^{1}$, Mikhail Dyagelev $^{1}$ and \\ Ivan Pushkarev ${ }^{1}$ \\ ${ }^{1}$ Kalashnikov Izhevsk state technical university, Studencheskaya 7, Izhevsk 426069, Russia
}

\begin{abstract}
The purpose of the work is to select the design concept of the wind power plant and the method of transformation of mechanical wind energy into electric energy depending on the wind speed in the given area. In order to solve the first task, it is proposed to approximate the distribution of wind speed with the help of the Raleigh Law, to use incomplete gamma functions and to compare the average annual power of wind plants with the vertical and horizontal spin axis. At low wind speeds dependence of wind plant mass with vertical spin axis with different types of generators is analyzed. The multiplication unit design is selected. Friction forces are determined in planetary multiplication unit having high efficiency. The effect of these forces on wind power plant efficiency is analyzed.
\end{abstract}

\section{Introduction}

The gradual depletion of fossil fuel deposits, the increase in the cost of fossil fuel extraction, the decrease in their availability and the transition to deposits in hard-to-reach areas have led to the need to turn to unconventional sources of energy. At present, due to their dispersion and variability, the latter cannot compete with hydrocarbon, atomic and hydraulic energy resources.

Small densities of renewable energy such as wind and solar energy lead to a significant increase in the area of energy receivers. In the case of wind power, low average wind speeds in Eurasia, excluding coastal areas, bring considerable difficulties [1]. As is known, wind power plants with a horizontal spin axis of the wind wheel, having high angular speeds of the rotor due to the special profile of the blades, start to operate only at some wind speed, usually more than $4 \mathrm{~m} / \mathrm{s}$. Wind power plants with a vertical spin axis are not limited from the bottom to start work, but today have a lower wind flow energy utilization factor $c_{p}$. In addition, the most convenient asynchronous motor requires high rotor speeds, and the windwheel with a vertical spin axis rotates at a low angular speed.

The purpose of the study is to determine the conditions under which it is advantageous to use a wind power plant design concept, including the type of wind wheel and the structure of the electromechanical part. It is proposed to solve the problems of selection: wind power plant design concept depending on the wind potential of the terrain; schemes

${ }^{*}$ Corresponding author: pushkarev@istu.ru 
for converting mechanical wind energy into electrical energy; type of multiplication unit with minimum mass-inertial characteristics and friction losses.

\section{Selection of design concept of wind power plant}

Wind speed is characterized by variability in time. Several wind speed distribution laws are used to estimate wind power potential. Knowing the law of distribution, it is possible to compare the efficiency of two options of wind power plants: with the vertical and horizontal spin axis. Average energy utilization of modern units can be taken equal to: with horizontal spun axis $c_{p}=0.42$, with vertical spin axis $c_{p}=0.36$.

A fairly good approximation to the actual measured wind speed data $u$ has a Raleigh distribution [2]:

$$
\Phi_{u}=(\pi / 2)\left(u / \bar{u}^{2}\right) \exp \left[-(\pi / 4)(u / \bar{u})^{2}\right],
$$

where $\Phi_{u}$ is probability of a given wind speed over a certain period of time (usually per year); $\bar{u}$ is average wind speed over that period of time.

In this case, the average wind power $\bar{N}$ can be expressed through the average speed in a given area:

$$
\bar{N}=c_{p}(\rho A / 2) \overline{u^{3}},
$$

where $\rho$ is air density; $A$ is area covered by wind wheel; $\overline{u^{3}}$ is average value of speed cube. By average value definition

$$
\overline{u^{3}}=\frac{\int_{0}^{\infty} \Phi_{u} u^{3} d u}{\int_{0}^{\infty} \Phi_{u} d u}=\frac{\pi}{2 \bar{u}^{2}} \int_{0}^{\infty} u^{4} \exp \left[-(\pi / 4)(u / \bar{u})^{2}\right] d u .
$$

The denominator in this expression is one. After substitution $v=(\pi / 4)(u / \bar{u})^{2}$ $\overline{u^{3}}=\frac{8 \bar{u}^{3}}{\pi \sqrt{\pi}} \int_{0}^{\infty} v^{3 / 2} \mathrm{e}^{-v} d v$. Здесь $\int_{0}^{\infty} v^{3 / 2} \mathrm{e}^{-v} d v=\Gamma\left(2+\frac{1}{2}\right)$ is gamma function. The partial value of the gamma function $\Gamma\left(n+\frac{1}{2}\right)=\sqrt{\pi} \frac{1 \cdot 3 \cdot 5 \ldots(2 n-1)}{2^{n}}$, from here $\Gamma\left(2+\frac{1}{2}\right)=\frac{3}{4} \sqrt{\pi}[3]$. Finally average speed cube value $\overline{u^{3}}=\frac{6}{\pi} \bar{u}^{3}$, power $\bar{N}=c_{p} \frac{3 \rho A}{\pi} \bar{u}^{3}$.

This mean power expression fully defines the mean power for a wind power plant with a vertical spin axis. Wind plants with horizontal axis start to operate only at some speed $u_{1}$ (Fig. 1), then, up to speed $u_{2}$, the average power value is by means of Raleigh distribution, then at wind speed $u_{2}$ the wind plant comes to nominal mode, and its power can be considered constant $N_{\text {nom }}$. 


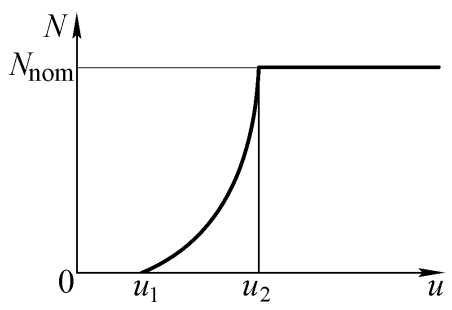

Fig. 1. Power of wind turbine unit with a horizontal spin axis

Average power of wind power plant with horizontal spin axis in the whole speed range:

$$
\bar{N}=c_{p} \frac{3 \rho A}{\pi} \int_{u_{1}}^{u_{2}} \Phi_{u} u^{3} d u+N_{\mathrm{H}} \Phi_{u>u_{2}}=c_{p} \frac{4 \rho A \bar{u}^{3}}{\pi \sqrt{\pi}} \int_{z_{1}}^{z_{2}} v^{3 / 2} \mathrm{e}^{-v} d v+c_{p} \frac{\rho A}{2} u_{2}^{3} \exp \left[-\frac{\pi}{4}\left(\frac{u_{2}}{\bar{u}}\right)^{2}\right],
$$

where $\Phi_{u>u_{2}}$ is probability of a velocity greater than $u_{2} ; z_{1}=(\pi / 4)\left(u_{1} / \bar{u}\right)^{2}$, $z_{2}=(\pi / 4)\left(u_{2} / \bar{u}\right)^{2}$ are new limits of integration. In terms of average power in the speed range $u_{1}-u_{2} \int_{z_{1}}^{z_{2}} v^{2.5-1} \mathrm{e}^{-v} d v$ is difference of two incomplete gamma functions $\gamma\left(\frac{5}{2}, z_{2}\right)=\int_{0}^{z_{2}} v^{2.5-1} \mathrm{e}^{-v} d v$ and $\gamma\left(\frac{5}{2}, z_{1}\right)=\int_{0}^{z_{1}} v^{2.5-1} \mathrm{e}^{-v} d v[2]$

Fig. 2 compares wind units with vertical and horizontal spin axis having different power utilization factors by specific power $N^{\prime}=\bar{N} /(\rho A)$.

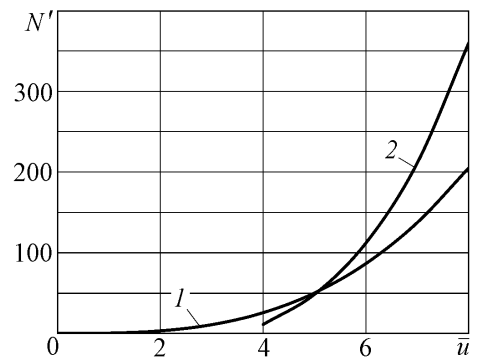

Fig. 2. Dependence of specific power of the wind turbine on the average speed of wind: 1 with a vertical spin axis; 2 with a horizontal spin axis

Thus, an analytical representation of the wind speed distribution makes it possible to estimate which wind plant design concept is more advantageous in terms of using wind potential. But the selection of the design concept of the entire wind power plant is also influenced by the parameters of the electromechanical part.

\section{Selection of method of energy transformation}

Constant speed of the windwheel is required when the wind unit is connected to the power grid $[4,5]$. Variable frequency uses a rectifier and inverter [6, 7]. Variable frequency is permissible when using wind energy for heat and water supply, for mechanical work. One possible use of a wind unit may be to drive the compressor of the heat pump. In the latter 
case, it is possible to significantly increase the basic operating index of the heat pump, in which the denominator is the operation required to compress the refrigerant.

There are two contradictions in the design of wind plants: the mismatch between the nominal angular speeds of the wind motor and the electric generator and the mismatch between the best operating modes of these two parts of the wind plant. Wind engine is usually low-speed machine with speed of 20-200 rpm, electric generator is high-frequency with speed of 1000-1500 rpm. The operation of the wind engine is effective in controlling the speed of its rotation in a large interval in which constant speed is ensured; a constant speed is preferred for the generator. There are two ways to solve these two contradictions.

The first is connection of wind engine with generator through multiplication unit (reduction gear). The second is connection of generator to power system by means of frequency converter.

Non-reductive direct-drive asynchronous or synchronous generator with speed of 20 200 rpm has considerable dimensions and weight. The multiplication unit compensates for this disadvantage. But, due to the introduction of another component in the system, the reliability of the system as a whole is reduced. However, careful consideration of mechanical operating conditions (frequent temperature changes, humidity, etc.) makes it possible to offset this disadvantage.

The choice of the version of the generator with multiplication unit or wuthout is also determined by the cost of the generator and the multiplication unit, which are related to the masses of these elements of the wind power plant. The weight and dimensions, in turn, depend on the torque and gear ratio. Fig. 3 shows the conditional dependence of the generator mass on the rotation speed, taking into account that the generator mass is inversely proportional to the frequency, as well as the dependence of the multiplication unit mass on the transmission ratio. The latter takes into account increase of required gear ratio of multiplication unit from increase of nominal generator frequency at constant low angular speed of wind wheel. The total mass, and therefore the cost of a wind power plant, has a hypothetical minimum.

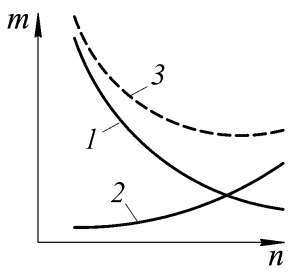

Fig. 3. Mass characteristics of wind power plant: 1 is generator weight; 2 is the weight of the multiplication unit; 3 is total weight of the whole plant

Considering the cost of the electric generator to some extent proportional to mass, as well as the increased complexity of the design, the circuit of the generator with the multiplication unit seems, in the foreseeable future, more expedient.

About $80 \%$ of modern wind plants are equipped with reduction gears (multiplication unit), which thus remain the most important part of modern wind power plants. A small proportion of low-power wind plants with generators on permanent magnets do not have multiplication units.

However, any multiplication unit connecting the windwheel axle to the generator increases the overall moment of inertia, thus making it difficult to drive the entire wind unit mechanism. Friction between the mechanism links also increases the kick-off torque. It is important to reduce the mass and moment of inertia of the rotating parts of the multiplication unit and to reduce friction power losses. 
From this point of view, the most promising gears for connecting the wind engine axle to the electric generator are planetary gears operating in multiplication mode. Planetary gears have the highest performance among other gears in terms of specific mass and power attributed to a unit of mass $[8,9]$.

Planetary gears are those with a roller torque absorption mechanism (Fig. 4) [9, 10].
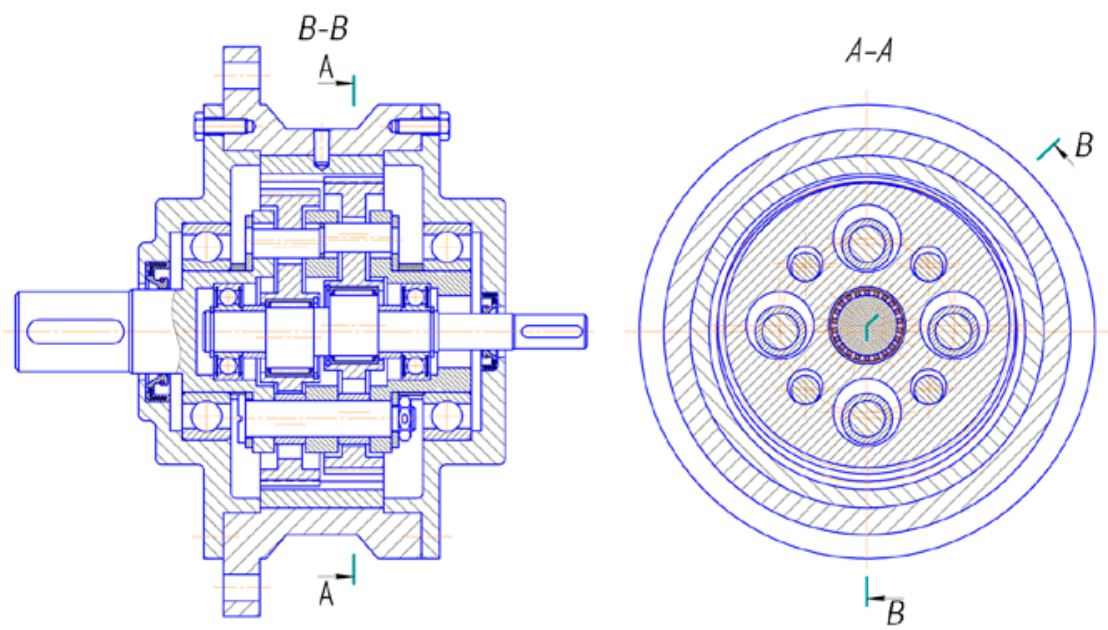

Fig. 4. Roller torque absorption gear

A significant advantage of the roller-teeth gear is the reduction of friction losses and, accordingly, the wind unit kick-off torque. Force analysis of this gear was performed in work [10]. A statically indeterminate problem is solved by the displacement method and expressions are found for normal forces $N_{j}$ in the case of the number of rollers $n=3,4,6$ and 8.

It is necessary to find out whether there is or is no sliding between rollers and holes in the satellite and the disc. No sliding will occur if the friction force does not exceed the limit friction force equal to the product of the friction coefficient by the normal force: $F=f N$. The design diagram for determining friction forces is shown in Fig. 5.

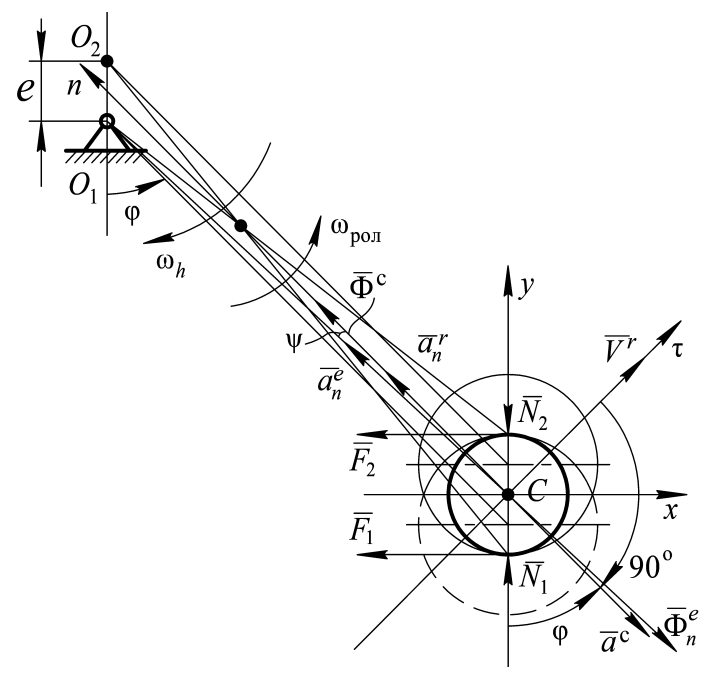

Fig. 5. Forces in roller-teeth gear 
Relative motion dynamics equation

$$
m \bar{a}_{n}^{r}=\bar{N}_{1}+\bar{N}_{2}+\bar{F}_{1}+\bar{F}_{2}+\bar{\Phi}_{n}^{e}+\bar{\Phi}^{c} ;
$$

where $\Phi_{n}^{e}=m \omega_{h}^{2} O_{1} C$ is force of moving space; $\Phi^{c}=2 m \omega_{h} V^{r} \sin 90^{\circ}=2 m \omega_{h} V^{r}$ is coriolis force; $m$ is mass of a roller; $\bar{a}_{n}^{r}$ и $V^{r}$ is relative acceleration and speed of the center of mass of the roller; $\omega_{h}$ is the angular speed of the carrier (windwheel axle).

From the rotational motion dynamics equation, assuming that there is no roller slip,

$$
I_{C} \varepsilon^{r}=\sum M_{C}=F_{2} r_{\text {roller }}-F_{1} r_{\text {roller }}=0
$$

follows that $F_{2}=F_{1}$.

Then

$$
2 F_{1}=m a_{n}^{r} \sin \varphi+m \omega_{h}^{2} r_{0} \sin \varphi-2 m \omega_{h} V^{r} \sin \varphi .
$$

After substituting known expressions and some transformations

$$
2 F_{1}=m \omega_{h}^{2} r_{0} \sin \varphi\left[\left(1+\frac{e}{r_{g}}\right)^{2} \frac{r_{\text {hole }}}{r_{\text {roller }}}+1-2\left(1+\frac{e}{r_{g}}\right)\right]=A m \omega_{h}^{2} r_{0}
$$

Where $A=\left(1+\frac{e}{r_{g}}\right)^{2} \frac{r_{\text {hole }}}{r_{\text {roller }}}+1-2\left(1+\frac{e}{r_{g}}\right) ; r_{0}$ is distance from the sateellite axis to the center of the hole in the satellite; $e$ is interaxle distance of roller-teeth gear; $r_{\text {hole }}$ is radius of the hole in the satellite and disk; $r_{\text {roller }}$ is roller radius; $r_{\text {hole }}=r_{\text {roller }}+(e / 2) ; r_{g}$ is satellite radius; $\varphi$ is the satellite angle of rotation.

Using the expressions for normal reactions found in [10], we obtain

$$
\frac{F_{1}}{N_{1}}=\frac{A m \omega_{h}^{2} r_{0}^{2}}{2 M}\left[\sin ^{2} \varphi+\sin ^{2}(\varphi+\tau)\right]
$$

where $\tau=2 \pi / n$ is the phase angle of the rollers; $M$ is the torque on the windwheel axle.

On any roller, the relationship of friction force and normal reaction is the same.

The friction force depends on the number of rollers $n$ :

$$
\begin{gathered}
n=3, \frac{F_{1}}{N_{1}}=\frac{A m \omega_{h}^{2} r_{0}^{2}}{2 M}\left[\sin ^{2} \varphi+\sin ^{2}\left(\varphi+\frac{2 \pi}{3}\right)\right] ; \frac{F_{1}}{N_{1}}=\frac{A m \omega_{h}^{2} r_{0}^{2}}{2 M} \\
n=4, \frac{F_{1}}{N_{1}}=\frac{A m \omega_{h}^{2} r_{0}^{2}}{2 M} ; n=6, \frac{F_{1}}{N_{1}}=\frac{3 A m \omega_{h}^{2} r_{0}^{2}}{4 M} ; n=8, \frac{F_{1}}{N_{1}}=\frac{A m \omega_{h}^{2} r_{0}^{2}}{M} .
\end{gathered}
$$

The relative friction force $F^{\prime}=\frac{2 M F_{1}}{A m \omega_{h}^{2} r_{0}^{2} N_{1}}$ is shown in Fig. 6 . 


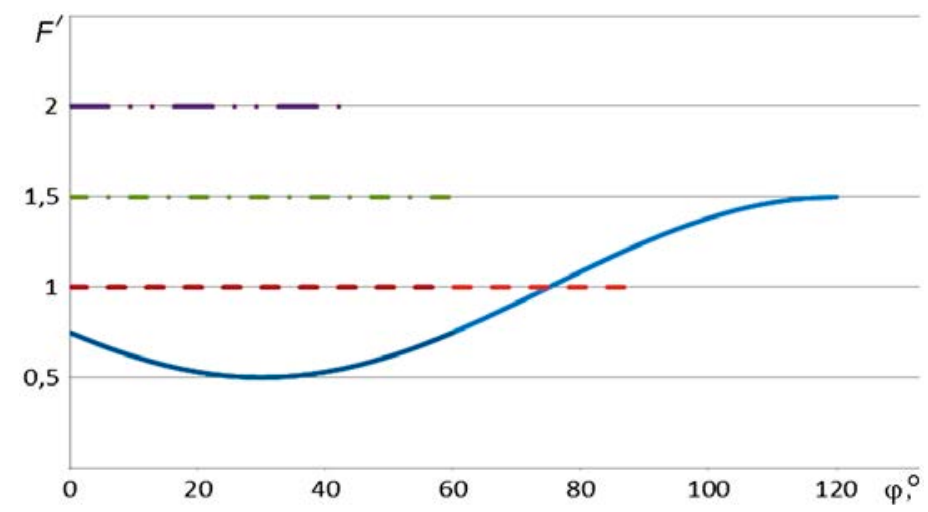

Fig. 6. Influence of number of rollers on friction force $F^{\prime}$ value: $\_3$ rollers; --4 rollers; - . 6 rollers; $\_$_ . 8 rollers

During one turn of the windwheel with an even number of rollers $n=4,6,8$, the ratio of friction force to normal reaction remains unchanged; at $n=3$ this ratio varying from 0.5 to 1.5 mean.

Taking values in expression (1) characteristic of wind unit with multiplication unit, $m=0.02-0.05 \mathrm{~kg} ; \omega_{h}=5-10 \mathrm{rad} / \mathrm{s} ; r_{0}=0.1-0.15 \mathrm{~m} ; M=10-20 \mathrm{Nm}$, ratio of friction force to normal reaction can be obtained, taking into account the number of rollers, within the range of $0.0001-0.01$, which is considerably less than friction coefficient, or ratio of limit friction force to normal reaction. Thus, the rollers in the roller-teeth planetary gear roll without sliding.

\section{Conclusions}

Based on the accepted Raleigh distribution of wind velocity probability is found that at power factors of wind plants with vertical and horizontal spin axis, respectively $c_{p}=0.36$ и $c_{p}=0.42$, the wind plant with vertical spin axis more efficiently uses wind flow energy in the range of low flow speeds. Starting with an average wind speed of about $5 \mathrm{~m} / \mathrm{s}$, a wind plant with a horizontal spin axis becomes more efficient. Thus, the analytical representation of the wind speed distribution allows to estimate which wind plant design concept is more advantageous in terms of using wind potential in a given area for a long time.

Taking into account the inversely proportional dependence of the generator mass on the rotation speed, and the growth of the multiplication unit mass with the increase in the transmission ratio, for wind power units with a vertical spin axis, having low angular speeds, it is more expedient to have an electromechanical scheme with a multiplication unit.

The most suitable gears for connecting the wind engine axle with the electric generator are planetary gears operating in multiplication mode. Significant reduction of friction in planetary multiplication unit of wind power plants can be achieved in roller-teeth gear. It has been proven that the rollers in the roller-teeth planetary gear roll without sliding, thereby replacing the sliding friction with rolling friction, increasing the efficiency of the planetary gear, and hence the entire wind power plant.

The work was carried out on a grant for scientists of the Kalashnikov Izhevsk State Technical University (project 08.04.01/18PAE). 


\section{References}

1. R. Hassanzadeh, Int. J. of Green Energy, V. 14, No. 2, 196-204 (2017)

2. J. Twidell, T. Weir, Renewable Energy Resources (London, New York, Routledge, 2015)

3. F. Olver, D. Lozier, R. Boisvert, C. Clark, NIST Handbook of Mathematical Functions (Cambridge University Press, 2010)

4. K. E. Okedu, IET Generation Transmission \& Distribution 13(15), 3378-3387 (2019)

5. M. Saeed, M.-H. Kim, Energy Conversion and Management, V. 134, 278-289 (2017)

6. S.F. Zhang, J. Yin, Y. Liu, Z.H. Sha, F.J. Ma, IOP Conf. Series, Mater. Sc. and Eng., ICMEAS 2016, 012026 (2016)

7. Y. Li, H. Li, P. Wang, Int. J. of Control and Automation, V. 9, No. 8, 359-368 (2016)

8. I. Bostan, V. Dulgheru, R. Ciobanu, IOP Conf. Series, Mater. Sc. and Eng., 7th ACME, 012002 (2016)

9. F. Plekhanov, V. Goldfarb, Mechanisms and Machine Science, V. 34, 285-300 (2016)

10. I. A. Pushkarev, T. A. Pushkareva, Proc. of Int. Sc. Conf. Math. Techniques in Engineering and Technology (MTET-31), V. 8, Ed A. A. Bolshakov (SanctPetersburg, SPBSTU), 40-43 (2018) 